\title{
Editorial
}

\section{New Strategies and Challenges in SHM for Aerospace and Civil Structures}

\author{
Alessandro Marzani, ${ }^{1}$ Zhongqing Su, ${ }^{2}$ and Ivan Bartoli ${ }^{3}$ \\ ${ }^{1}$ Department of Civil, Chemical, Environmental and Materials Engineering, University of Bologna, 40126 Bologna, Italy \\ ${ }^{2}$ Department of Mechanical Engineering, The Hong Kong Polytechnic University, Hung Hom, Kowloon, Hong Kong \\ ${ }^{3}$ Department of Civil, Architectural and Environmental Engineering, 3141 Chestnut Street, Philadelphia, PA 19104, USA \\ Correspondence should be addressed to Alessandro Marzani; alessandro.marzani@unibo.it
}

Received 18 February 2013; Accepted 18 February 2013

Copyright (c) 2013 Alessandro Marzani et al. This is an open access article distributed under the Creative Commons Attribution License, which permits unrestricted use, distribution, and reproduction in any medium, provided the original work is properly cited.

This special issue includes six original research papers discussing new approaches and laboratory validations as well as practical field applications of structural health monitoring (SHM) techniques for aerospace, mechanical, and civil structures. A variety of methodologies including guided waves, finite element model updating, and vibration-based methods are presented. A brief description of each contribution is provided below.

A team of researchers from the University of Bologna (Italy) presented a nonlinear signal processing approach based on a warped frequency transform (WFT) to compensate for the dispersive behavior of ultrasonic guided waves, followed by a Wigner-Ville time-frequency analysis and a Hough transform to further improve the defect detectability and localization accuracy of Lamb wave inspection systems. As a result, an automatic detection procedure to locate defectinduced reflections was demonstrated and successfully tested by analyzing numerically simulated Lamb waves propagating in an aluminum plate.

A different group of researchers from the University of Bologna (Italy) discussed the structural health monitoring of an ancient railway masonry arch bridge located in Bologna. In particular, they developed a three-dimensional finite element model of the entire bridge and updated leveraging data from an in situ experimental campaign involving static and dynamic load tests performed on the structure. The study included also material testing performed on samples of the masonry structure extracted from the bridge. The structural performances of the bridge both in its actual state and in the case of a retrofitting action were assessed.
A research group consisting of researchers from Shandong Agricultural University (China), Hohai University (China), the Polish Academy of Sciences (Poland), and The Hong Kong Polytechnic University (Hong Kong) proposed a fractal dimension-based analysis of mode shapes for damage identification of beam structures. In particular, they were able to obviate false peaks of high magnitude in fractal dimension due to the natural inflexions of higher-order mode shapes by using specially designed affine transformations, thus overcoming limitations of classical fractal-based methods. The new method was numerically demonstrated on a multiple cracked cantilever beam and was experimentally validated using a scanning laser vibrometer to acquire higher-order mode shapes.

A team from the Northwestern Polytechnical University (China) and The Hong Kong Polytechnic University (Hong Kong) presented a paper on the estimation of the probability density function of measured data extracted from Lamb wave-based damage detection procedures in the presence of strong measurement noise. In particular, they proposed and validated a nonparametric method based on the kernel density estimation in order to enhance the estimation of the probability density function. The results showed that the nonparametric methods outperformed the empirical methods in terms of accuracy.

A group from the University of California, San Diego (USA), presented a work on the generation of higher harmonics in waveguides of complex cross-section. The paper extended classical semianalytical finite element formulations to the nonlinear regime analysis for predicting second- 
harmonic generations. Nonlinear guided waves represent an attractive phenomenon for structural health monitoring since they exhibit large sensitivity to structural damage compared to traditional approaches based on linear wave features. Results were presented for a railroad track and a viscoelastic plate. For the investigated cases, optimum combinations of primary wave modes and resonant doubleharmonic nonlinear wave modes have been identified.

Finally, a group from the Shanghai Jiao Tong University (China) reported a three-dimensional spectral element method (SEM) for the analysis of Lamb wave propagation in composite laminates containing delamination. SEM is more efficient than conventional finite element method (FEM) in simulating wave propagation in structures because of its unique diagonal form of the mass matrix. The effectiveness of the method was validated by comparing the simulation results with analytical solutions based on the transfer matrix method. By a parametric analysis it was demonstrated that symmetric Lamb waves can be insensitive to delamination at certain interfaces of laminates while the antisymmetric waves are more suited for identification of delamination in composite structures.

Alessandro Marzani Zhongqing Su Ivan Bartoli 


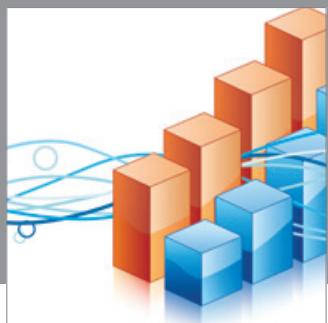

Advances in

Operations Research

mansans

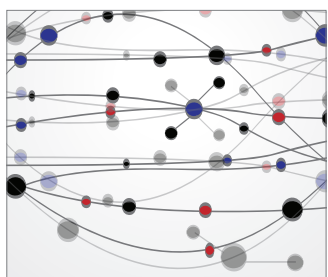

The Scientific World Journal
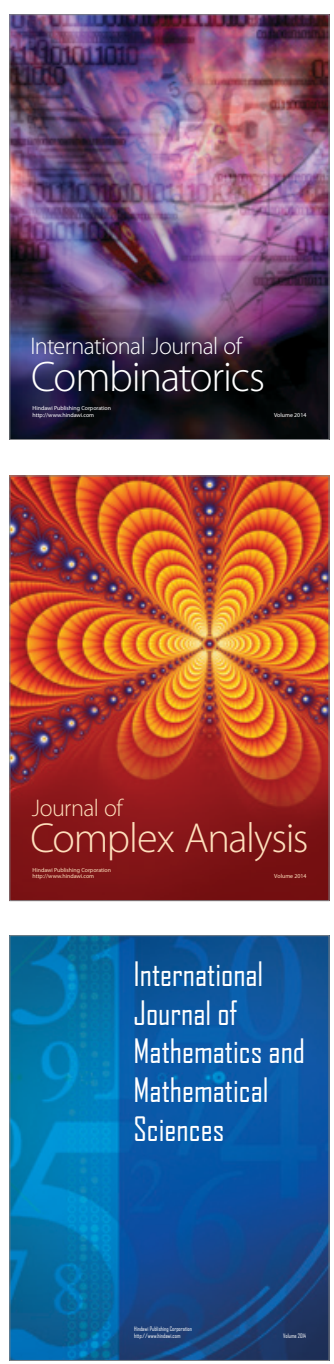
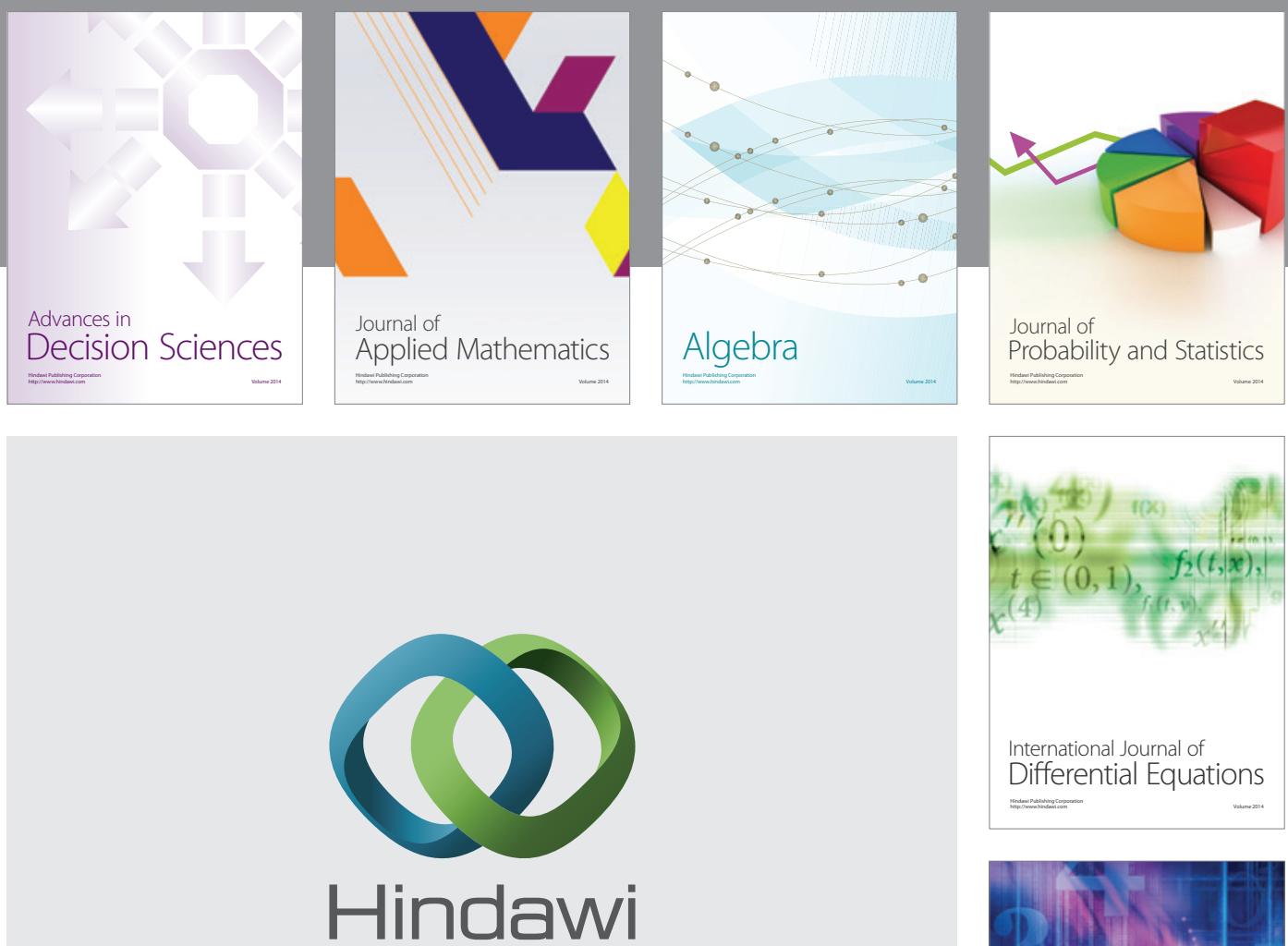

Submit your manuscripts at http://www.hindawi.com
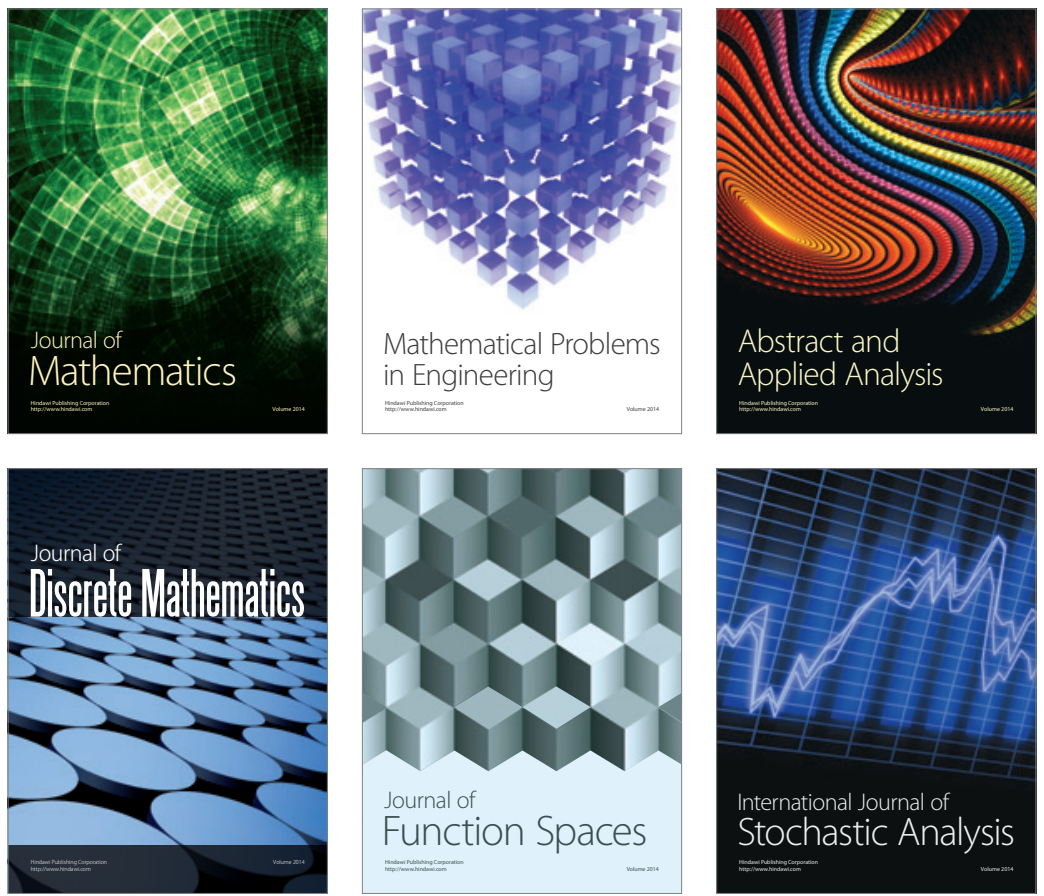

Journal of

Function Spaces

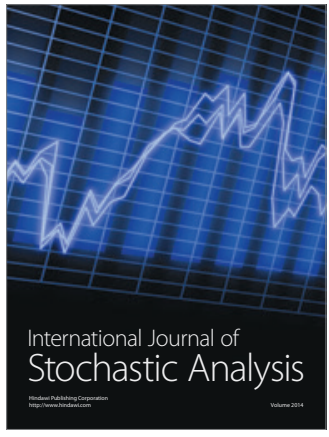

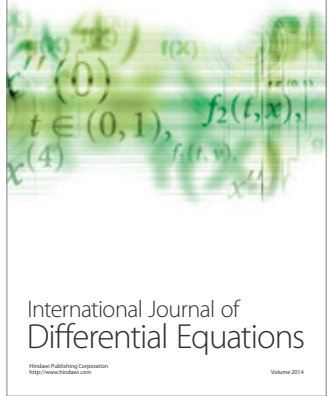
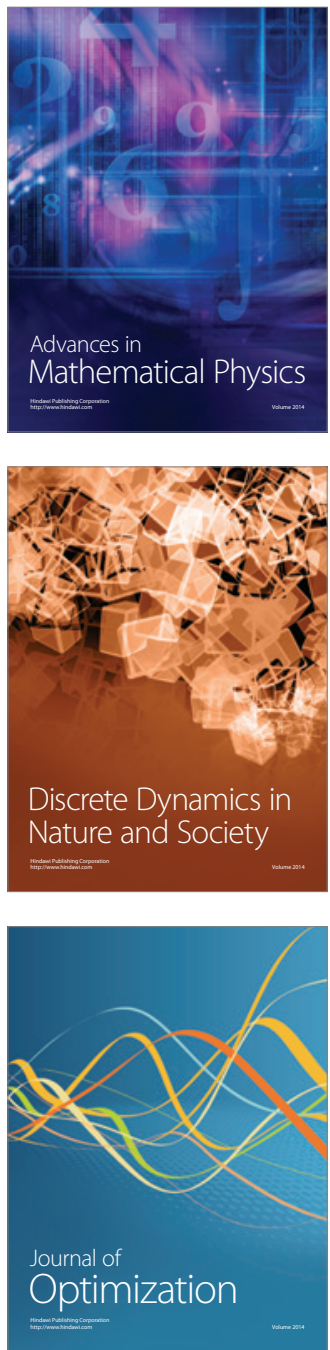